Research Paper

\title{
Vector-Mediated Selective Expression of Lethal Factor, a Toxic Element of Bacillus Anthracis, Damages A549 Cells via Inhibition of MAPK and AKT Pathways
}

\author{
Wenlei Zhuo ${ }^{1,}$, Guangli Tao ${ }^{2}$, Liang Zhang ${ }^{3}$, Zhengtang Chen ${ }^{1}$ \\ 1. Institute of Cancer, Xinqiao Hospital, Third Military Medical University, Chongqing, China; \\ 2. Department of Nephrology, Xinqiao Hospital, Third Military Medical University, Chongqing, China; \\ 3. Department of Environmental Hygiene, College of Preventive Medicine, Third Military Medical University, Chongqing, China.
}

$\triangle$ Corresponding author: Wenlei Zhuo, E-mail: zhuowenlei@yahoo.com.cn. Institute of Cancer, Xinqiao Hospital, Third Military Medical University, Chongqing 400038, China.

( ) Ivyspring International Publisher. This is an open-access article distributed under the terms of the Creative Commons License (http://creativecommons.org/ licenses/by-nc-nd/3.0/). Reproduction is permitted for personal, noncommercial use, provided that the article is in whole, unmodified, and properly cited.

Received: 2012.1I.21; Accepted: 2013.01.25; Published: 2013.01.27

\begin{abstract}
Lethal factor (LF), a major toxic element of Bacillus anthracis combined with its protective antigen (PA), enters the cells through the cytomembrane receptors and causes damage to the host cells, thereby leading to septicemia, toxemia, and meningitis with high mortality. LF has been identified as a potential biotech-weapon, which can impede cancer growth in vascular endothelial cells because of its cytotoxicity. However, the feasibility of LF application and further investigations has been limited because LF is nonspecific. To solve this problem, we constructed a vector that contained the LF sequence, which was regulated by a tumor-specific human telomerase reverse transcriptase promoter ( $\mathrm{hTERTp}$ ). Results showed that LF was selectively expressed in lung cancer A549 cells but not in normal cells, thereby resulting in A549 cell apoptosis. The results also revealed that the inhibition of mitogen-activated protein kinase and AKT pathways was partially involved in the process. Thus, hTERTp-regulated LF increase could be a promising approach in lung cancer-targeted therapy.
\end{abstract}

Key words: Anthrax lethal factor; Selective expression; Targeted therapy; A549 cell; Signaling pathway.

\section{Introduction}

Lung cancer is one of the leading causes of cancer-related mortality worldwide, in which non-small cell lung cancer (NSCLC) accounts for about $80 \%$ of all cases and $40 \%$ of NSCLCs are in an advanced stage at the time of diagnosis.

Chemotherapy and radiotherapy have been applied as the main treatment strategies for NSCLCs for several years. However, the efficacy of both treatments has been weakened by chemoresistance and radioresistance of most NSCLCs. Thus, new methods for treating NSCLCs should be developed. Gene therapy is a promising approach.
Bacillus anthracis, the causative agent of anthrax, is a spore-forming Gram-positive bacterium and the pathogen of acute anthrax disease in humans and animals. Systemic B. anthracis infection is characterized by septicemia, toxemia, and meningitis, which is the main neurological complication associated with high mortality [1]. B. anthracis consists of three proteins, including protective antigen (PA), lethal factor (LF), and edema factor (EF). PA combines with LF to form LTx, a major toxic factor that elicits cytotoxicity and translocates LF into the cells through the cytomembrane receptors. Evidence has suggested that 
LTx has more significant functions in pathogenesis than ET [2]. LF disrupts mitogen-activated protein kinase (MAPK) signaling by cleaving the upstream mitogen-activated protein kinase kinases (MEKs). Thus, it has been regarded as a cellular toxin [3]. Hence, LF may be a promising biotech-weapon for cancer therapy.

Previous studies assessed the efficacy of LF on cancer cells by combining LF and PA at a specific proportion. LF can significantly inhibit the cell growth of cancers, such as melanoma [4], fibrosarcoma [5], renal cancer [6], and lung cancer [7]. Moreover, LF can disrupt the endothelial cells [8] and significantly control tumor angiogenesis $[9,10]$. Although the cytotoxic LF and PA combination eradicates the cancer cells, this combination also damages the normal cells because it is nonspecific. Therefore, the application of LF on cancer research has been limited. To address this concern, a new treatment method should be developed.

One possible solution involves increasing LF expression in tumor cells without affecting the normal cells via genetic techniques. Tumor-targeted vectors that are regulated by tumor-specific promoters can specifically express therapeutic genes in the tumor cells. Evidence has suggested that the human telomerase reverse transcriptase promoter (hTERTp), which contains a high G/C content without TATA or CAAT box, is highly expressed in most human cancer cells, including lung cancer, but not in normal cells [11]. Therefore, hTERTp has been considered a tumor-specific promoter in cancer-targeted therapy [12]. In this study, we hypothesized that hTERTp could specifically stimulate LF expression in lung cancer cells. Reports have also shown that antibodies against PA can neutralize lethal toxin $[13,14]$, indicating that LF cannot enter the normal cells without PA to elicit the toxic effects after the host cancer cells have collapsed.

In this study, we aimed to construct an hTERTp-regulated plasmid that carried the LF sequence and determine whether or not the specific LF expression could selectively damage A549 cells. We also assessed the possible involvement of MAPK and AKT signaling pathways in this process.

\section{MATERIALS AND METHODS}

\section{Plasmid construction}

pAAV-MCS plasmid vectors (Stratagene, USA) were used for vector-based DNA synthesis. To obtain genomic DNA clones that contained hTERTp, the cDNA product for hTERTp was synthesized according to Horikawa et al. [15]. MluI (CGCGT) and BsaBI
(GATTG) restriction enzyme cutting sites were respectively added to the 5'- and $3^{\prime}$-end of the first strand: F: 5'-CGCGTGACGCCCAGGACCGCGCTT CCCACGTGGCGGAGGGACTGGGGACCCGGGCA CCCGTCCTGATTG-3' and R: 3'-ACTGCGGGTC CTGGCGCGAAGGGTGCACCGCCTCCCTGACCCC TGGGCCCGTGGGCAGGACTAAC-5' . For the LF sequence, the full length of the LF gene was searched through the Gene Library (M30210). The coding sequence (CDS; $2331 \mathrm{bp}$ ) was selected and synthesized. Xbal (CTAGA) and SacI (GAGCT) restriction enzyme cutting sites were added to the $5^{\prime}$ - and $3^{\prime}$ - end of the first strand, respectively. The sequences were synthesized and cloned successfully.

pAAV-MCS was simultaneously digested by $M l u I$ and BsaBI exogenous enzymes. The CMV promoter (CMVp) was then removed. The remaining part of the plasmid was subsequently connected and further amplified with hTERTp by T4 ligase. The plasmid was extracted according to the manufacturer's protocol. The phTERTp-MCS was obtained and digested by Xbal and SacI to be linearized. Afterward, phTERTp-MCS was linked to the LF sequence and the linkage was amplified. A phTERTp-LF plasmid that contained the upstream LF sequence with hTERTp was successfully constructed. pAAV-MCS was cut by $\mathrm{Xbal}$ and SacI, linked to the LF sequence, and cloned. The pCMV-LF plasmid that contained the CMVp-stimulated LF was constructed.

\section{Cell culture and transfection}

The human lung adenocarcinoma cell line, A549 cell line, and the human fetal lung fibroblast (MRC5) were obtained from the American Type Culture Collection and conserved in the Central Laboratory of Xinqiao Hospital. The cells were cultured in Dulbecco's Modified Eagle's Medium (DMEM, HyClone, Logan, UT) that contained $5 \%$ fetal bovine serum in a humidified atmosphere with $5 \% \mathrm{CO}_{2}$ at $37^{\circ} \mathrm{C}$ and transfected using the lipofectamine plus transfection reagent (Invitrogen, Carlsbad, CA) with the vectors according to the manufacturer's protocol.

\section{Cell viability assay}

For quantitative viability assays, the cells were plated in 96-well plates $\left(1 \times 10^{4}\right.$ cells/well). 3-(4,5-Dimethylthiazol-2-yl)-2,5-diphenyl tetrazolium bromide (MTT) assays were performed to assess the cell viability. Sterile MTT dye $(200 \mu \mathrm{l}$; $5 \mathrm{mg} / \mathrm{ml}$; Sigma, USA) was added. After the cells were incubated for $4 \mathrm{~h}$ at $37^{\circ} \mathrm{C}$ in $5 \% \mathrm{CO}_{2}$, the MTT medium mixture was removed and $200 \mu \mathrm{l}$ of dimethyl sulfoxide was added to each well. Absorbance was determined at $490 \mathrm{~nm}$ by using a multi-well spectro- 
photometer (Thermo Electron, Andover, USA).

\section{Cell apoptosis analysis}

Apoptotic cells were evaluated using an annexin V-FITC kit (Beyotime, China). The cells were scraped and stained with annexin V-FITC and propidium iodide according to the manufacturer's protocol. In brief, the cells were washed with PBS. After $195 \mu \mathrm{l}$ of the binding buffer was added, $5 \mu 1$ of FITC-labeled annexin $\mathrm{V}$ was added and incubated for $10 \mathrm{~min}$ at 25 ${ }^{\circ} \mathrm{C}$. The cells were then incubated with 10 $\mu l$ propidium iodide for $10 \mathrm{~min}$ in an ice bath in the dark and the apoptotic cells were determined by flow cytometry (FACS) analysis.

\section{RT-PCR assay}

Total RNA was isolated using the TRI Reagent (Molecular Research Center, Cincinnati, $\mathrm{OH}$ ) and the first-strand cDNA was synthesized from $1 \mu \mathrm{g}$ of total RNA by using oligo $d(T)$ primer and ReveTra Ace (TOYOBO, Osaka, Japan). The cDNA product was amplified by polymerase chain reaction (PCR) with the following primers for LF (197 bp): F, 5'-GAAACATCGGTCTGGAAATA-3' and R, 5'-ATGCACGTTGAATGTAATAAG-3'; for GAPDH (452 bp), 5'-ACCACAGTCCATGCCATCAC-3', 5'-TCCACCACCCTGTTGCTGTA-3'. The thermal cycler parameters used were as follows: one cycle at $94{ }^{\circ} \mathrm{C}$ for $30 \mathrm{~s} ; 30$ cycles of denaturation at $94{ }^{\circ} \mathrm{C}$ for 30 $\mathrm{s}$ and annealing at $50^{\circ} \mathrm{C}$ for $30 \mathrm{~s}$ with an extension at $72{ }^{\circ} \mathrm{C}$ for $60 \mathrm{~s}$. The extension was performed for another $10 \mathrm{~min}$ after the indicated cycles were completed. The bands were quantified by densitometric scanning of the band intensities and normalized to glyceraldehyde-3-phosphate dehydrogenase (GAPDH) levels by using the Image-Pro Plus 5.0 software (Media Cybernetics, Silver Spring, MD, USA).

\section{Western blot analysis}

The cells were harvested, pelleted by centrifugation, washed with ice-cold PBS, and lysed with RIPA buffer [ $150 \mathrm{mM} \mathrm{NaCl}, 50 \mathrm{mM}$ Tris base ( $\mathrm{pH} 8.0), 1 \mathrm{mM}$ EDTA, $0.5 \%$ sodium deoxycholate, 1\% NP-40, 0.1\% sodium dodecyl sulfate (SDS), $1 \mathrm{mM}$ DTT, $1 \mathrm{mM}$ PMSF, and $1 \mathrm{mM} \mathrm{Na} 3 \mathrm{VO}_{4}$ ] that was supplemented with a protease inhibitor. The proteins were separated by $10 \%$ SDS-polyacrylamide gel electrophoresis and transferred onto a nitrocellulose membrane (Life Technologies, Gaithersburg, MD). The blots were then incubated in a fresh blocking solution with an appropriate dilution of the primary antibody at $4{ }^{\circ} \mathrm{C}$ for 24 $h$. The sources of antibodies were as follows: LF goat polyclonal antibody and GAPDH goat polyclonal antibody (Santa Cruz); p-MAPK (Thr202/Tyr204), MAPK, and p-AKT (Ser473); and AKT rabbit monoclonal antibody (Cell Signaling). After the blots were extensively washed, the membranes were incubated with horseradish peroxidase-coupled secondary antibody (1:2000, Zhongshan Biotech Company, China) at $25^{\circ} \mathrm{C}$ for $1 \mathrm{~h}$. The bands were visualized and quantified using the Image-Pro Plus 5.0 software (Media Cybernetics). p-MAPK and p-AKT band intensities were normalized to MAPK and AKT band intensities, respectively. LF intensities were adjusted according to the GAPDH band intensities.

\section{Statistical analysis}

Data were expressed as mean value \pm SD. Differences between groups were analyzed using ANOVA or a $t$-test. These analyses were performed on SPSS version 13.0 for Windows (SPSS Inc., Chicago, IL). $P$-value $<0.05$ was considered statistically significant.

\section{Results}

\section{hTERTp selectively induced LF expression}

The pAAV-MCS vector contains CMVp and other elements for high-level gene expression in mammalian cells when a specific gene is cloned into multiple cloning sites. In this study, we constructed two vectors that contained LF-coding sequences. In pCMV-LF, the LF sequence was stimulated by CMVp. In phTERTp-LF, CMVp was replaced with hTERTp.

We divided A549 and MRC5 cells into three groups. The cells were treated with phTERTp-LF, pCMV-LF, and DMEM for $24 \mathrm{~h}$. Six types of cells were then created: A549-nontransfection, A549-hTERTpLF, A549-CMV-LF, MRC5-nontransfection, MRC5-hTERTp-LF, and MRC5-CMV-LF cells. RNA that was extracted from the transfected cells was subjected to RT-PCR analysis. Figure 1A shows that a significant increase in LF mRNA expression was found in A549-hTERTp-LF, A549-CMV-LF, and MRC5-CMV-LF cells but not in MRC5-hTERTp-LF, MRC5-nontransfection, and A549-nontransfection cells, suggesting that the vector-mediated transfected cells, which contained hTERTp, could selectively induce LF expression in cultured cancer cells. In A549 cells, phTERTp-LF and pCMV-LF vectors could significantly increase the LF mRNA expression. In MRC5 cells, pCMV-LF performed this function. 


\section{A}
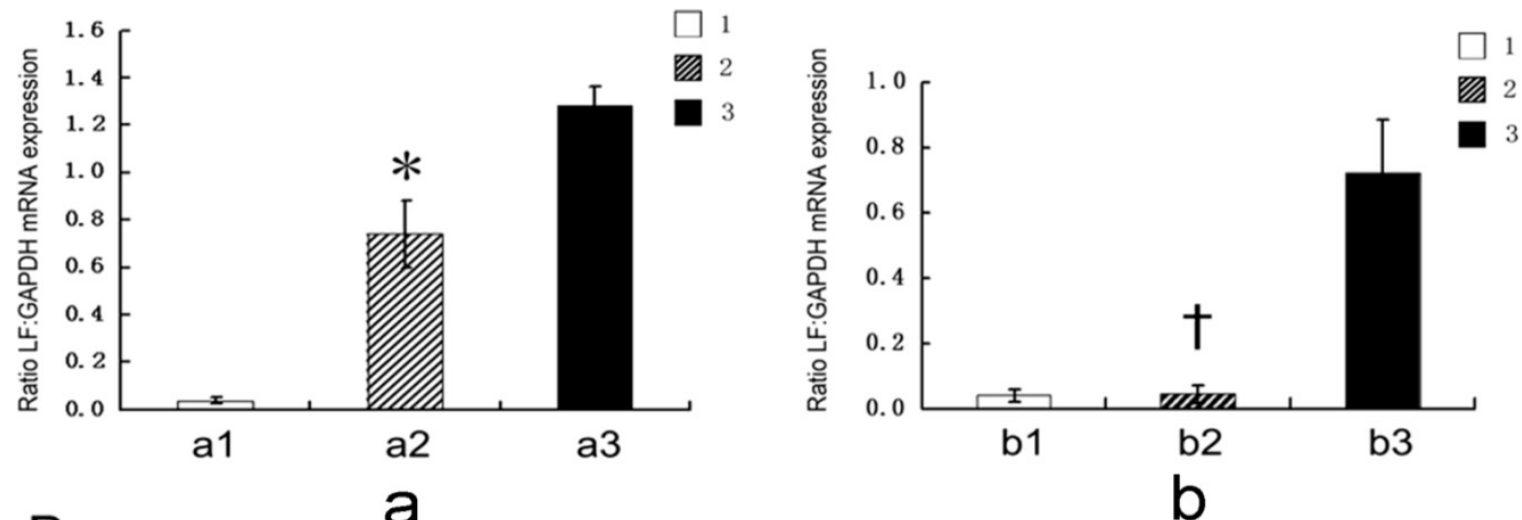

\section{B}

a
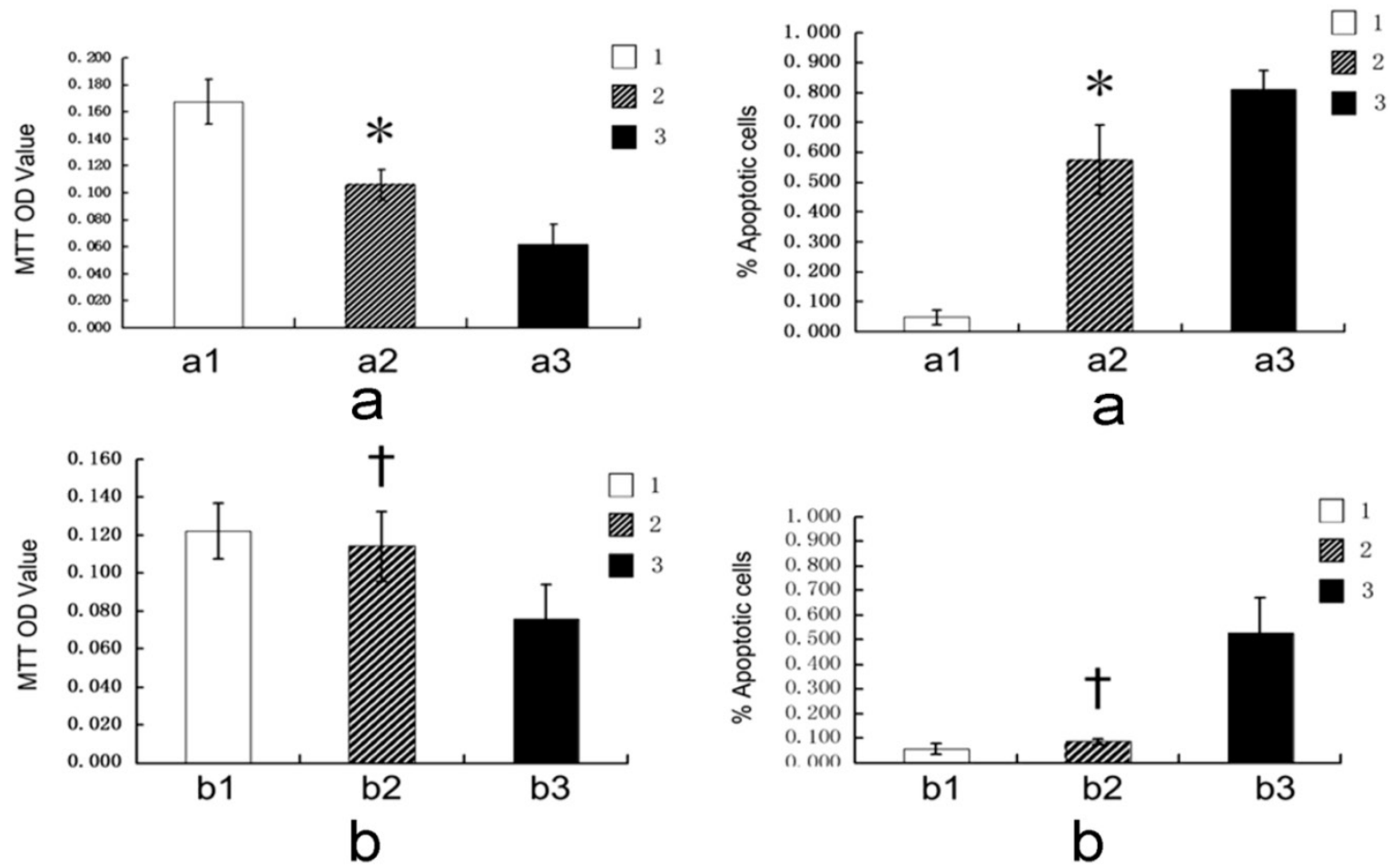

Figure I. (A) Expression of LF mRNA in A549 (a) and MRC5 (b) cells (* $P<0.05$ vs al or a3; $\nmid P<0.05$ vs b3; $\nmid P>0.05$ vs bl). (B) Cell viability and apoptosis in A549 (a) and MRC5 (b) cells assessed by MTT and apoptosis assays $(* P<0.05$ vs al or a3; $\nmid P<0.05$ vs b3; $\nmid P$ $>0.05$ vs bl). I. DMEM; 2. phTERTp-LF; 3. pCMV-LF (al. A549-nontransfection; a2. A549-hTERTp-LF; a3. A549-CMV-LF; bl. MRC5-nontransfection; b2. MRC5-hTERTp-LF; b3. MRC5-CMV-LF ).

\section{hTERTp-induced LF gene expression in- creased apoptosis of A549 cells but not MCS-5 cells}

To investigate the possible functions of LF in A549 cells, the cells were assessed by MTT and apoptosis assays, respectively. The results showed that the increase in LF expression resulted in a significant decrease in cell viability and an increase in apoptosis of A549 and MRC5 cells (Figure 1B). The results also indicated that LF expression may induce apoptosis in tumor and normal cells. However, hTERTp-stimulated LF could selectively impair the tumor cells but not the normal control cells, indicating that hTERTp may be a promising tumor-specific promoter in lung cancer-targeted therapy.

\section{LF expression increased A549 cell apoptosis possibly by inactivating MAPK and AKT pathways}

The underlying mechanisms by which the selective LF expression damages A549 cells remain un- 
clear. Increasing evidence has indicated that several signaling pathways are possibly involved in this process.

To determine the signaling pathways that could be involved, MAPK, p-MAPK, AKT, and p-AKT were further assessed by immunoblotting. The results showed a significant decrease in p-MAPK and p-AKT expressions in A549-hTERTp-LF, A549-CMV-LF, and MRC5-CMV-LF cells compared with that in MRC5-hTERTp-LF, MRC5-nontransfection, and A549-nontransfection cells (Figure 2), suggesting that the inhibition of MAPK and AKT signaling pathways may be involved in LF-mediated cell apoptosis.

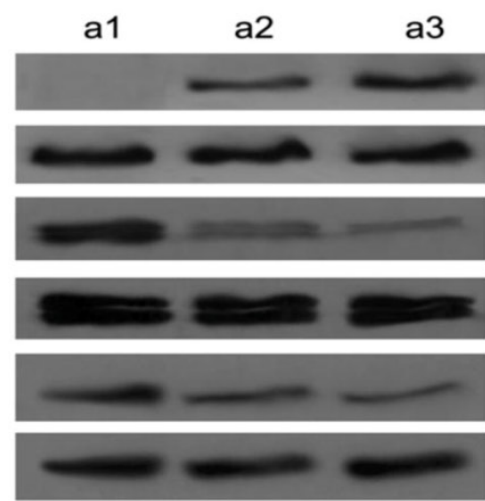

a

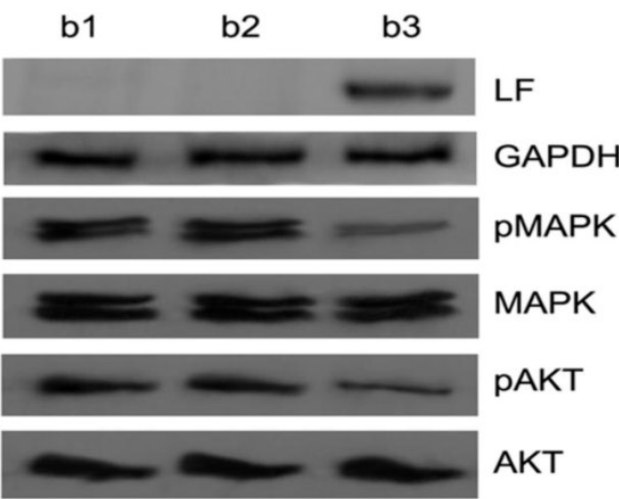

b
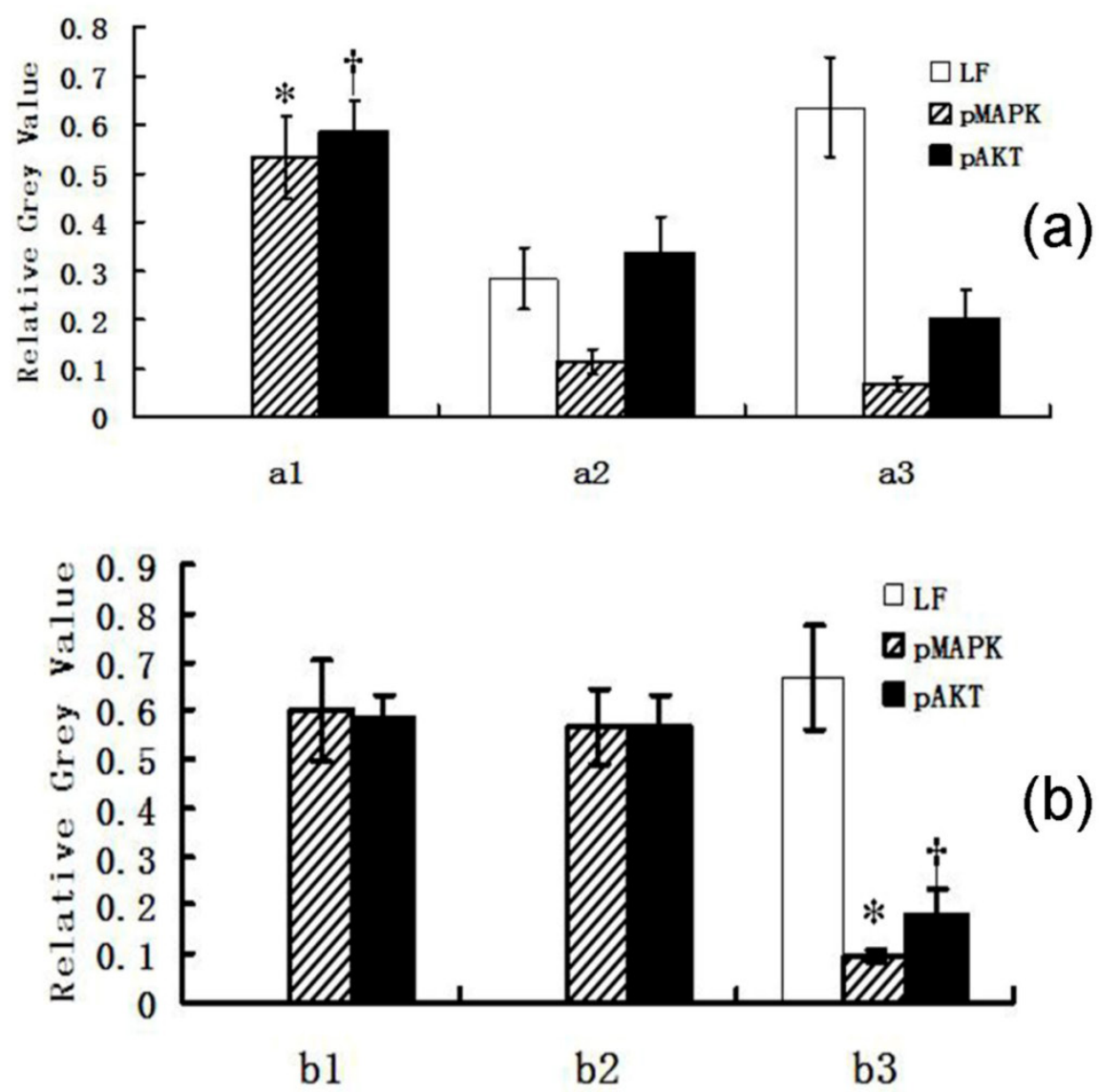

Figure 2. Expression of LF, MAPK and AKT pathway-related proteins in A549 (a) and MRC5 (b) cells assessed by Western blot (al. A549-nontransfection; a2. A549-hTERTp-LF; a3. A549-CMV-LF; bI. MRC5-nontransfection; b2. MRC5-hTERTp-LF; b3. MRC5-CMV-LF ). The average relative gray value is calculated from three independent blots and presented as mean \pm S.D. (a): $* P<0.05$ vs a2 or a3; $\uparrow P<0.05$ vs a2 or a3; (b) $* P<0.05$ vs bl or b2; $\nmid P<0.05$ vs bl or b2. 

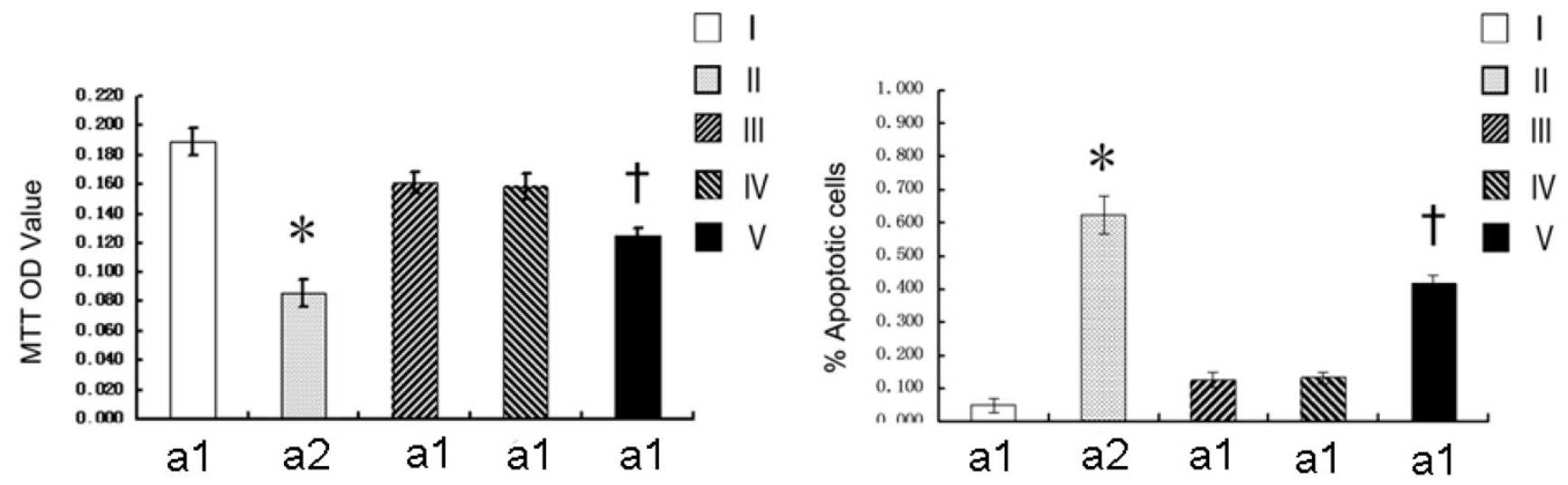

Figure 3. Cell viability and apoptosis of cells treated with DMEM (I), DMEM (II), U0 I 26 (III), MK-2206 (IV), U0 I 26+MK-2206 (V) assessed by MTT and apoptosis assay, respectively. ( $P<0.05$ vs I or III; $\nmid P<0.05$ vs I or II). (a I): A549-nontransfection; (a2): A549-hTERTP-LF.

\section{Inactivation of MAPK and AKT pathway may be another possible pathway of LF-mediated cell apoptosis}

To determine the possible functions of MAPK and AKT pathways, we divided the cells into five groups (I, II, III, IV, and V). Group I had A549-nontransfection cells that were treated with DMEM for $6 \mathrm{~h}$ and used as the control group. For group II, A549-hTERTp-LF cells were treated with DMEM for $6 \mathrm{~h}$. The A549-nontransfection cells in groups III, IV, and V were pre-treated with $10 \mu \mathrm{M}$ of U0126 (a specific MAPK inhibitor, Sigma), $10 \mu \mathrm{M}$ of MK-2206 (a specific AKT inhibitor, Sigma), and a combination of $10 \mu \mathrm{M}$ of $\mathrm{U} 0126$ and $10 \mu \mathrm{M}$ of MK-2206 for $1 \mathrm{~h}$, respectively. The A549-nontransfection cells in groups III, IV, and V were also treated with DMEM for $6 \mathrm{~h}$. Cell viability and apoptosis were assessed by MTT and apoptosis assays, respectively.

U0126 and MK-2206 are established inhibitors of MAPK and AKT phosphorylation, respectively. The pre-treatment with U0126 (III) or MK-2206 (IV) slightly induced A549-nontransfection cell apoptosis compared with the untreated A549-nontransfection (I). Moreover, the pretreatment with U0126 and MK-2206 (V) significantly induced A549-nontransfection cell apoptosis compared with that in group III or IV. However, this effect $(\mathrm{V})$ was slightly different from that in A549-hTERTp-LF cells in group II (Figure 3).

\section{Discussion}

To the best of our knowledge, this study was the first to describe the functions and possible mechanisms by which vector-based selective LF expression induces hTERTp-stimulated A549 cell apoptosis.

Researchers $[16,17]$ used the combination of PA and LF to assess the possible functions of LF on cancers and found that the cytotoxicity of LF may be potentially applied in cancer therapy. Nevertheless, LF enters the normal cells via PA and exhibits cytotoxicity. To resolve this problem, we used the vectors with the LF sequence that was selectively expressed in the lung cancer A549 cells in the present study. Our results showed that the LF expression significantly induced cell apoptosis of A549 cells without affecting the normal cells.

hTERTp transcription is activated in cancer cells but is repressed in normal primary cells. Hence, hTERTp is used as a tumor-specific promoter that specifically expresses therapeutic genes in tumor tissues. In this study, CMVp could effectively induce LF expression in A549 and MRC5 cells. This finding indicated that CMVp is nonspecific. By contrast, hTERTp successfully induced LF expression only in A549 cells, indicating that hTERTp could selectively induce LF expression in cancer cells.

MAPKs, which mainly comprise ERK, p38MAPKs, and c-Jun N-terminal kinase, possibly function in mediating cell apoptosis. LF cleaves MEKs and enzyme-phosphorylating MAPKs, and thus has been associated with the apoptosis of several kinds of cells $[18, \mathbf{1 9}]$. AKT, a serine/threonine protein kinase, has an important function in multiple cellular processes, such as cell proliferation, apoptosis, transcription, and cell migration. Evidence has indicated that AKT, which may be associated with lung epithelial cell apoptosis, may be inhibited by $B$. anthracis infection [20]. LF can significantly cleave MEKs, thereby inactivating the MAPK signaling pathway, inducing cell death, and suppressing angiogenesis [21]. The present study confirmed that the MAPK signaling pathway was inhibited after the vector was transfected. The AKT signaling pathway was also inhibit- 
ed. However, the results indicated that these pathways may partially function in the cytotoxicity of LF in A549 cells. In this process, the activation of MAPK and AKT signaling pathways provided a survival signal, whereas the inhibition of both pathways released a partial apoptotic signal, indicating that multiple pathways in addition to MAPK and AKT signaling pathways may be involved in this process. Thus, further investigations should be conducted regarding the possible pathways that could be involved in the process.

Several limitations were observed in this study. First, we used A549 cell line, a well-characterized human lung adenocarcinoma cell line that has been widely used for lung cancer experimental research. Further research on other cell lines may strengthen the significance of our data, which indicated that hTERTp-stimulated LF expression was detected in lung cancer A549 cells rather than in normal cells. Second, this study only focused on the functions and the possible mechanisms of LF expression in vitro, in which the selective expression of LF may be a possible biotech-weapon against A549 cells and a promising strategy for lung cancer therapy. Thus, further in vivo studies are needed to determine the specific mechanisms and evaluate the feasibility of LF gene therapy.

In conclusion, we constructed an hTERTpstimulated plasmid-containing LF gene that successfully damaged the lung cancer cells through cell transfection without affecting the normal cells. Thus, the inhibition of MAPK and AKT signaling pathways may be partly involved in the process and increased the possibility that the vector-mediated, hTERTp-regulated selective LF expression may be a promising approach in lung cancer-targeted therapy.

\section{Conflict of Interest}

None declared.

\section{Acknowledgements}

This work was supported by the Chongqing Natural Science Foundation (NO:CSTC, 20085124), National Natural Science Foundation of China (NSFC) grant (No:30800506) and Scientific research innovation fund of Third Military Medical University (No:2008D204).

\section{Competing Interests}

The authors have declared that no competing interest exists.

\section{References}

1. Ebrahimi CM, Sheen TR, Renken CW, Gottlieb RA, Doran KS. Contribution of lethal toxin and edema toxin to the pathogenesis of anthrax meningitis. Infection and immunity. 2011; 79: 2510-8. doi:10.1128/IAI.00006-11.

2. Pezard C, Berche P, Mock M. Contribution of individual toxin components to virulence of Bacillus anthracis. Infection and immunity. 1991; 59: 3472-7.

3. Mourez M. Anthrax toxins. Reviews of physiology, biochemistry and pharmacology. 2004; 152: 135-64. doi:10.1007/s10254-004-0028-2.

4. Abi-Habib RJ, Singh R, Leppla SH, Greene JJ, Ding Y, Berghuis B, et al. Systemic anthrax lethal toxin therapy produces regressions of subcutaneous human melanoma tumors in athymic nude mice. Clinical cancer research : an official journal of the American Association for Cancer Research. 2006; 12: 7437-43. doi:10.1158/1078-0432.CCR-06-2019.

5. Ding Y, Boguslawski EA, Berghuis BD, Young JJ, Zhang Z, Hardy K, et al. Mitogen-activated protein kinase kinase signaling promotes growth and vascularization of fibrosarcoma. Molecular cancer therapeutics. 2008; 7: 648-58. doi:10.1158/1535-7163.MCT-07-2229.

6. Huang D, Ding Y, Luo WM, Bender S, Qian CN, Kort E, et al. Inhibition of MAPK kinase signaling pathways suppressed renal cell carcinoma growth and angiogenesis in vivo. Cancer research. 2008; 68: 81-8. doi:10.1158/0008-5472.CAN-07-5311.

7. Kong D, Li Y, Wang Z, Banerjee S, Sarkar FH. Inhibition of angiogenesis and invasion by 3,3'-diindolylmethane is mediated by the nuclear factor-kappaB downstream target genes MMP-9 and uPA that regulated bioavailability of vascular endothelial growth factor in prostate cancer. Cancer research. 2007; 67: 3310-9. doi:10.1158/0008-5472.CAN-06-4277.

8. Liu T, Milia E, Warburton RR, Hill NS, Gaestel M, Kayyali US. Anthrax lethal toxin disrupts the endothelial permeability barrier through blocking p38 signaling. Journal of cellular physiology. 2011. doi:10.1002/jcp.22859.

9. Alfano RW, Leppla SH, Liu S, Bugge TH, Duesbery NS, Frankel AE. Potent inhibition of tumor angiogenesis by the matrix metalloproteinase-activated anthrax lethal toxin: implications for broad anti-tumor efficacy. Cell Cycle. 2008; 7: 745-9.

10. Cryan LM, Rogers MS. Targeting the anthrax receptors, TEM- 8 and CMG-2, for anti-angiogenic therapy. Frontiers in bioscience : a journal and virtual library. 2011; 16: 1574-88.

11. Fujiwara T, Tanaka N. Telomerase-specific oncolytic virotherapy for human cancer with the hTERT promoter. Uirusu. 2008; 58: 11-8.

12. Deng WG, Jayachandran G, Wu G, Xu K, Roth JA, Ji L. Tumor-specific activation of human telomerase reverses transcriptase promoter activity by activating enhancer-binding protein-2beta in human lung cancer cells. The Journal of biological chemistry. 2007; 282: 26460-70. doi:10.1074/jbc.M610579200.

13. Crowe SR, Garman L, Engler RJ, Farris AD, Ballard JD, Harley JB, et al. Anthrax vaccination induced anti-lethal factor IgG: fine specificity and neutralizing capacity. Vaccine. 2011; 29: 3670-8. doi:10.1016/j.vaccine.2011.03.011.

14. Reason D, Liberato J, Sun J, Camacho J, Zhou J. Mechanism of Lethal Toxin Neutralization by a Human Monoclonal Antibody Specific for the PA(20) Region of Bacillus anthracis Protective Antigen. Toxins. 2011; 3: 979-90. doi:10.3390/toxins3080979.

15. Horikawa I, Cable PL, Afshari C, Barrett JC. Cloning and characterization of the promoter region of human telomerase reverse transcriptase gene. Cancer research. 1999; 59: 826-30.

16. Frankel AE, Koo HM, Leppla SH, Duesbery NS, Vande Woude GF. Novel protein targeted therapy of metastatic melanoma. Current pharmaceutical design. 2003; 9: 2060-6.

17. Rozanov DV, Golubkov VS, Strongin AY. Membrane type-1 matrix metalloproteinase (MT1-MMP) protects malignant cells from tumoricidal activity of re-engineered anthrax lethal toxin. The international journal of biochemistry \& cell biology. 2005; 37: 142-54. doi:10.1016/j.biocel.2004.06.005.

18. Bluthgen N, Legewie S. Systems analysis of MAPK signal transduction. Essays in biochemistry. 2008; 45: 95-107. doi:10.1042/BSE0450095.

19. Kim EK, Choi EJ. Pathological roles of MAPK signaling pathways in human diseases. Biochimica et biophysica acta. 2010; 1802: 396-405. doi:10.1016/j.bbadis.2009.12.009.

20. Popova T, Espina V, Bailey C, Liotta L, Petricoin E, Popov S. Anthrax infection inhibits the AKT signaling involved in the E-cadherin-mediated adhesion of lung epithelial cells. FEMS immunology and medical microbiology. 2009; 56: 129-42. doi:10.1111/j.1574-695X.2009.00558.x.

21. Kirby JE. Anthrax lethal toxin induces human endothelial cell apoptosis. Infection and immunity. 2004; 72: 430-9. 